\title{
Bodies and their parts
}

\author{
JULIE BAUER MORRISON \\ Bryant University, Smithfield, Rhode Island \\ and \\ BARBARA TVERSKY \\ Stanford University, Stanford, California
}

\begin{abstract}
How do we think about the space of bodies? Several accounts of mental representations of bodies were addressed in body part verification tasks. An imagery account predicts shorter times to larger parts (e.g., back $<$ hand). A part distinctiveness account predicts shorter times to more discontinuous parts (e.g., arm < chest). A part significance account predicts shorter times to parts that are perceptually distinct and functionally important (e.g., head $<$ back). Because distinctiveness and significance are correlated, the latter two accounts are difficult to distinguish. Both name-body and body-body comparisons were investigated in four experiments. In all, larger parts were verified more slowly than smaller ones, eliminating the imagery/size account. Despite the correlation between distinctiveness and significance, the data suggest that when comparisons are perceptual (body-body), part distinctiveness is the best predictor, and when explicit or implicit naming is involved, part significance is the best predictor. Naming seems to activate the functional aspects of bodies.
\end{abstract}

All of our lives, we live inside our bodies. The view we have of bodies, then, is privileged; we experience bodies from inside as well as from outside. We have internal sensorimotor knowledge of bodies, in addition to the external sensory knowledge that we have of other objects. Our internal experience of bodies is an intricate web, including proprioceptive stimulation, feedback from our own activities, conscious experience, and intentions. Does our insider's knowledge of our own body affect our understanding of bodies?

A variety of evidence from anatomy, lesions, stimulation, imaging, and behavior suggests that knowledge of the body is privileged, giving support for the elusive concept of body schema. According to Parsons's (1990) description, a body schema is an, "abstract internal representation of spatial and physical-mechanical properties of one's body. It is based on some combination of past and current information ...., as well as from our sense of physical effort and from contact with objects and among our body parts" (p. 46).

What characterizes the body schema? For one thing, it seems to incorporate kinesthetic or kinematic knowledge. Parsons (1994) speculated that kinematic mental rotation of the body, rather than visual mental rotation, underlies right-left judgments of hands depicted in many

This research was supported in part by Office of Naval Research Grants N00014PP10649, N000140110717, and N000140210534 to Stanford University. The authors thank Elizabeth Marsh, Pamela Schraedley, and Mija Van Der Wege for their assistance with the statistical analyses. Correspondence concerning this article should be addressed to J. B. Morrison, Department of Psychology, Glendale Community College, 6000 W. Olive Avenue, Glendale, AZ 85302 (e-mail: julie.morrison@gcmail.maricopa. edu). orientations. Both reaction times and imaging data support the claim (Parsons, 1994; Zacks, Mires, Tversky, \& Hazeltine, 2000; Zacks, Ollinger, Sheridan, \& Tversky, 2002).

Body kinematics also affects the perception of body positions (Reed \& Farah, 1995). While moving their arms or legs, observers viewed pairs of complex body poses photographed from different angles, to decide whether poses, same or differing either in the arms or the legs, were the same or different. Moving the arms enhanced detection of arm differences, whereas moving the legs enhanced detection of leg differences. Overall, differences in upper bodies were detected better than differences in lower bodies. Body movement was selective to body recognition; body movement did not affect judgments of upper or lower differences in Lego towers.

Determination of differences in body positions is facilitated, then, by actual body movement. The potential for body movement affects the perception of apparent motion (Chatterjee, Freyd, \& Shiffrar, 1996; Shiffrar \& Freyd, 1990). Observers saw pairs of pictures of bodies or objects in rapid succession, a procedure that typically elicits apparent motion. The shortest path of motion would require seeing a part of an object pass through the body or the object. For bodies, at interstimulus intervals that were relatively long, but short enough to yield apparent motion, observers reported seeing longer paths of motion that were congruent with a biomechanical movement, going around the body, rather than through it. This did not occur for objects, even though movement of one object through another is physically unlikely.

Neuropsychological and neurological evidence suggests that body knowledge is encapsulated to some degree. One patient suffered severe left personal neglect; 
nevertheless, his schema of the space beyond his body was unimpaired (Guariglia \& Antonucci, 1992). Functional fMRI has shown that a region in the lateral occipitotemporal cortex, referred to as the extrastriate body area, or EBA, selectively responds to visual images of human bodies and body parts, but not to whole faces, objects, object parts, or scrambled bodies (Downing, Jiang, Shuman, \& Kanwisher, 2001). Presumably, such activation would facilitate perception and action if the expected part actually received stimuli.

Early work on disorders of the body schema identified the parietal lobes as the locus of integration of various information about the body (e.g., Roth, 1949). More recently, Gross and Graziano (1995) have proposed that the posterior parietal cortex is the center of spatial processing, bringing together touch, vision, and proprioception. Specialized areas important to spatial functioning - for example, navigation and memory of external space - are all part of the network controlled by the posterior parietal cortex.

These are but a few of the findings that converge to suggest that knowledge of the body is relatively independent and that it includes proprioceptive, kinesthetic, and kinematic information. Would this knowledge be reflected in perceptual and cognitive tasks, such as body part verification? Body parts differ on a number of characteristics, including size, distinctiveness, and function. Some of these features are primarily visual, whereas others depend on sensorimotor information. Which factors account for the time required to verify common body parts? We will consider three theories: one from imagery, one from object recognition, and the third from object categorization.

The part size account grows out of the classic literature on imagery. Starting from the assumption that large things are perceived more rapidly, Kosslyn $(1976,1980)$ proposed that large parts of images should be detected more quickly than smaller ones. He found that participants verified large parts, such as the back of a rabbit, more quickly than smaller ones, such as the ears, when the participants verified parts from an image. Applied to the context of verifying body parts, this account would predict that large parts, such as the back and the leg, should be verified more quickly than smaller parts, such as the hand and the foot.

The part distinctiveness account is an extension of research on object recognition - specifically, shape theories based on parts (Biederman, 1987; Hoffman \& Richards, 1984). These theories propose that objects are recognized by recognizing their parts. According to these accounts, part decomposition occurs at inflection points, or points of greater discontinuity, along the object contour. Applying this account to bodies would predict that parts with greater contour discontinuity or distinctiveness, such parts as the head and the foot, should be verified more quickly than such parts as the chest and the back.

A third account, part significance, derives from research on the organization of object categories. Accord- ing to that work, people use perceptually salient parts to infer part function (Tversky \& Hemenway, 1984). Parts of common objects, both animate and inanimate, that were rated highest on goodness had both functional significance and perceptual salience - for example, the legs of a chair or a dog. Perceptual salience was essentially determined by contour discontinuity. A part significance account would predict that body parts higher in functional significance and perceptual salience would be verified more quickly. More significant body parts are those with greater sensory and motor representation, relative to their size; this would enable greater motor agility and greater sensory sensitivity. An index of part significance, then, would be size in the sensorimotor cortical map, relative to physical size. The often depicted homunculus in the postcentral gyrus for somatosensory information and in the precentral gyrus for motor information shows that some relatively smaller body parts, such as the hand, have larger cortical representation, relative to their size, than some larger parts do, such as the back (Penfield \& Rasmussen, 1950). These findings correspond with two-point skin thresholds, which are relatively smaller for body parts such as the head, the hand, and the foot than for relatively larger parts, such as the leg and the back (Weinstein, 1968). On the basis of part significance, highly significant parts such as the head and the hand, which are overrepresented in the sensorimotor cortex, relative to their actual size, should have shorter verification times than do less significant parts, such as the back and the leg, which are underrepresented in the sensorimotor cortex, relative to their size. Another index of part significance is people's ratings, to be described.

Note that the predictions from part distinctiveness and part significance are similar. This is not surprising, since part significance has inputs both from perceptual salience, which correlates with part discontinuity, and from functional significance. On the whole, part distinctiveness and part significance are highly correlated. These two accounts do differ in predicting verification times for specific pairs of parts. For the body parts used here, the chest has less contour discontinuity than the leg or the foot does, but has greater significance. The chest is in the upper half of the body; it represents the front, the most important side of the body (see, e.g., Franklin \& Tversky, 1990), and it shelters many of the important internal body parts - the heart and lungs, for example.

Here, we explore the roles of part size, distinctiveness, and significance in body part verification tasks of two types: body-body comparisons and name-body comparisons. We chose body parts that are likely to be important, reflected by the fact that they are named with single lexemes across many languages (Brown, 1976). Including only a small number of popular parts severely restricts the range for correlations. The body parts selected - the head, arm, hand, chest (front), back, leg, and foot-were those that are most commonly named across cultures (Andersen, 1978; Brown, 1976; Burton \& Kirk, 1979) and those that are more or less in the same size scale. The 
selected parts were also those that children all over the world include in their early drawings of people: a small circle over a larger one, with four sticks protruding, each with smaller protrusions (Goodnow, 1977; Kellogg, 1969). Thus, there is good reason to believe that these body parts are regarded as important.

Because the part distinctiveness and part significance accounts make nearly identical predictions, the main goal of these experiments was to compare the size account with the other accounts. The evidence separating the distinctiveness and the significance accounts will be more suggestive than definitive. The reason for the two types of comparisons is that names of body parts may emphasize their meanings but depictions may emphasize their perceptual features. Especially in tasks such as this one, with repeated trials for a small set of similar stimuli, responding to purely visual stimuli may become automatized, so that pictures of bodies may be treated without regarding them as such.

\section{EXPERIMENT 1}

\section{Simultaneous Body-Body Part Verification}

In this experiment, participants viewed two depictions of human bodies, each with one body part cued. Their task was to respond same if the cued body parts were the same and to respond different otherwise. The question of interest was the time taken to verify the different body parts. These were compared with the results of a questionnaire asking other participants to rank body parts according to size, distinctiveness, or significance.

\section{Method}

Participants. Twenty-six Stanford University students participated in this experiment for course credit. The data from 7 participants were eliminated due to error rates above $10 \%$. Analyses were conducted on the data from 11 women and 8 men.

Stimuli. Twelve different poses of realistic-looking human bodies were created using the Fractal Design Poser (1995) software, 2 for training and 10 for testing (see Figure 1). In this experiment, 8 of the 10 testing poses were used. Two poses, stand and squat, the most redundant with the other 8 , were eliminated in order to decrease the length of this experiment but were included in some of the following experiments. Pose names were used for design purposes only and were not mentioned to the participants.

Poses were selected to represent a broad, realistic, and possible range of human postures. All the poses were in profile in order to eliminate left/right judgments, with facing direction counterbalanced across poses. The poses were created to maximize the amount and types of body part comparisons, as well as to maximize the possible distance between various body parts. Each body was shown in three orientations: $0^{\circ}, 90^{\circ}$, and $180^{\circ}$. Previous research has suggested that as objects and bodies are rotated further from upright, recognition times increase (Carpenter \& Just, 1978; Cooper, 1975; Cooper \& Shepard, 1973; McMullen \& Jolicœur, 1992; Metzler, 1973; Parsons, 1987; Tarr \& Pinker, 1991). Unlike earlier research, in the experiments reported here, small increments of rotation were not used, making it unlikely that the same types of rotation effects would be observed. Rotating the poses served to provide diversity in the stimuli. It also provided an extra level of difficulty for the task, resulting in a more meaningful processing of the stimuli. The

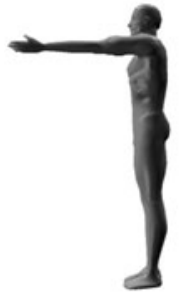

Stop

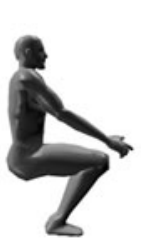

Hands-on-Knees

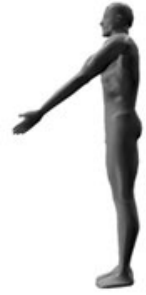

Stand

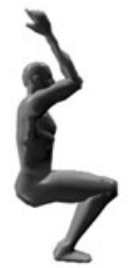

Squat

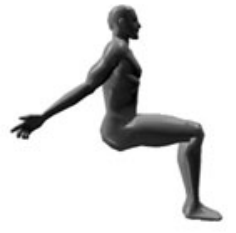

Reverse Hands-on-Knees
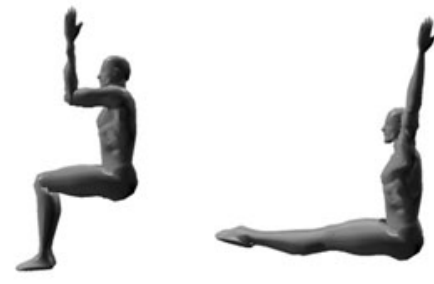

Up/Front

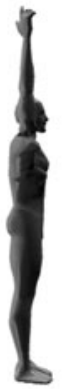

Point

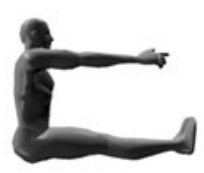

Stretch

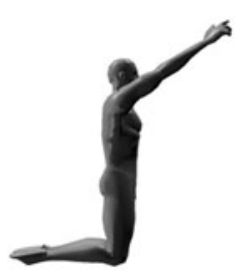

Reach

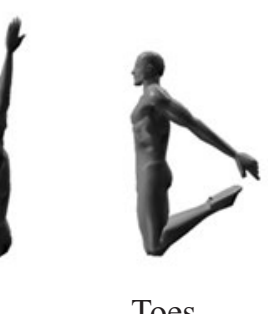

Toes

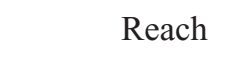

Figure 1. Body poses. Shown here are the 12 body poses used during experimentation, 10 during testing and 2 during training (reverse hands-on-knees and point back). In Experiments $3 B$ and 4 , re-creations of the poses from an updated and more realistic version of Fractal Design Poser (1996) were used. Testing poses stand and squat were eliminated from Experiments 1 and 4.

rotated poses were interpreted as rotated pictures of bodies, not as bodies in those orientations, as evidenced by the finding that upright poses, both standing and sitting, were responded to most quickly (see Experiment 3A). This suggests that participants, when interpreting upright bodies, assume a support system, such as a floor or a chair. Finally, we varied the orientation of the entire body, rather than varying the pose, because comparing different poses on a single trial adds the variance of differences in the positions of individual body parts, relative to entire bodies. 
Figure 2 shows the seven body parts cued on each body, using a uniform-sized white dot placed approximately in the center of the body part. For the point and up/front poses, where the arm obscures part of the head, the dot was placed closer to the nose. The seven cued parts were the head, arm, hand, chest, back, leg, and foot.

Each image showed two bodies in the same pose, but at different orientations (see Figure 3). There were three combinations of orientation possible per trial: $0^{\circ}$ and $90^{\circ}, 0^{\circ}$ and $180^{\circ}$, and $90^{\circ}$ and $180^{\circ}$, with left/right position counterbalanced.

Design. Fourteen questions were asked of each body at each orientation pair, seven same questions and seven different. For the same questions, cued body parts matched. Of the 21 different body part combinations possible, 7 were used four times in each of the 10 poses, and the remaining 14 combinations were used three times.

Equipment. This study was run on Apple Power Macintosh $7200 / 75$ computers with 17-in. AppleVision monitors. The experiment was designed and run with PsyScope (1994) software. Reactions times were measured to millisecond accuracy, using the PsyScope button box. The button box contains three keys: red, yellow, and green. Labels placed above the keys identified the red button as different, the green button as same, and the yellow button as next. Lefthanded participants had the option of reversing the same and different buttons so that their dominant hand provided the same response.

Procedure. On each trial, the participants saw a fixation point centered on the screen for $500 \mathrm{msec}$, followed by the pair of bodies. When the participant pressed the same or the different key in response to the bodies, they heard a beep, the poses disappeared, and they were instructed to press a third key to continue. The participants completed the 18-trial training session, during which they were encouraged to ask questions; then they completed 336 test trials at their own pace. Randomized versions of the test trials were presented to the participants. The study took approximately $30 \mathrm{~min}$ to complete.

\section{Results and Discussion}

Although for this and the subsequent experiments we analyzed the data from both same and different responses, only the data from analyses of same responses will be reported in detail. The comparison of same and different

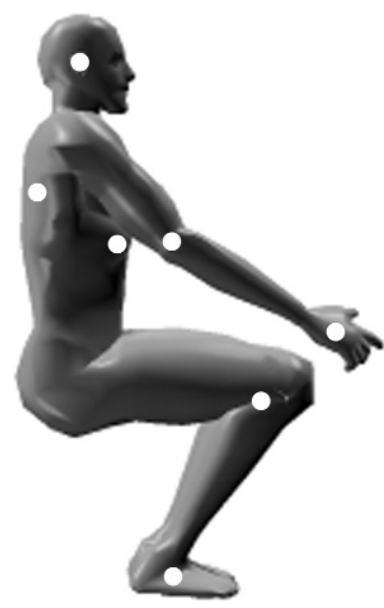

Figure 2. Cued body parts. This pose depicts the seven cued body parts used during this study: the head, the arm, the hand, the chest, the back, the foot, and the leg. Only one part would have been cued on each body during a trial. responses is not of interest here, and the same responses give a cleaner estimate of body part verification times. Since there were no consistent overall effects of particular pose or of gender of the participant, these analyses will not be reported as well.

Across all the experiments reported here, errors and reaction times greater than two standard deviations from the participant's mean were removed from the data. The task was a simple one, so errors or long response times reflect either a misstroke or a lack of concentration on a particular trial. Because of the repetitive nature of the task, the participants were instructed that they could pause during the experiment by delaying their response, yielding extreme response times. Across all the experiments, removed data accounted for no more than $7.5 \%$ of all the responses, with approximately $3 \%$ errors and $4 \%$ outliers. A small number of participants, those who had over $10 \%$ of their data removed, were excluded altogether from the analyses.

A significance level of $p<.05$ was adopted for the analyses of variance (ANOVAs). Bonferroni corrections were applied to all paired $t$ tests, resulting in a significance level of $p<.017$ when three variables were involved (e.g., orientation $t$ tests), $p<.005$ when five variables were involved (Experiment 2, body part analyses), and $p<.002$ when seven variables were involved (all the other body part analyses).

For same responses, we performed a two-way repeated measures ANOVA with within-subjects factors of body part (arm, back, chest, foot, hand, head, or leg) and orientation disparity $\left(90^{\circ}\right.$ or $\left.180^{\circ}\right)$, the degree of rotation difference between the two poses. The angular disparity between the two poses did not affect the response times $\left[F(1,18)=0.12, M S_{\mathrm{e}}=2,893, p=.74\right]$. There was a part $\times$ orientation interaction, such that responses to some body parts were slower at $180^{\circ}$ disparity than at $90^{\circ}$ $\left[F(6,108)=4.24, M S_{\mathrm{e}}=5,512, p<.01\right]$.

Figure 4 shows that the pattern of verification times for the seven body parts was shortest for the head $(M=$ $979 \mathrm{msec}, S D=298)$, followed by the hand $(M=$ $1,000 \mathrm{msec}, S D=291)$, the foot $(M=1,046 \mathrm{msec}, S D=$ $311)$, the leg $(M=1,044 \mathrm{msec}, S D=300)$, the arm $(M=1,112 \mathrm{msec}, S D=327)$, the chest $(M=1,158 \mathrm{msec}$, $S D=352)$, and the back $(M=1,260 \mathrm{msec}, S D=344)$ $\left[F(6,108)=46.31, M S_{\mathrm{e}}=8,302, p<.01\right.$. There were sizable differences between the response times for the various parts. Head times were shorter than those for all the other parts, except for the hand [arm, $t(18)=6.68$, $p<.001$; back, $t(18)=12.46, p<.001$; chest, $t(18)=$ $7.24, p<.001$; foot, $t(18)=4.81, p<.001$; leg, $t(18)=$ $5.69, p<.001]$. Back times were longer than those for all the other parts [arm, $t(18)=6.21, p<.001$; chest, $t(18)=5.33, p<.001$; foot $t(18)=10.21, p<.001$; hand, $t(18)=10.16, p=.001$; leg, $t(18)=11.17, p<$ $.001]$, whereas arm and chest times were longer than those for the head, the chest, the hand [arm, $t(18)=$ $4.70, p<.001$; chest, $t(18)=5.82, p<.001]$, the foot 

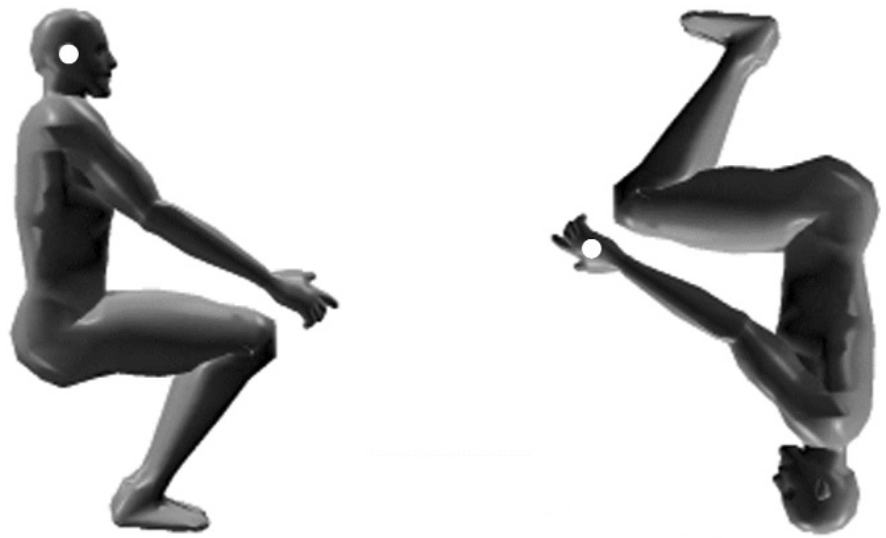

Figure 3. Body-body stimuli. The participants saw two matching poses and verified whether the cued parts were the same or different. Poses were presented at orientations of $0^{\circ}, 90^{\circ}$, or $180^{\circ}$, with a difference of $90^{\circ}$ or $180^{\circ}$ between the two poses.

[arm, $t(18)=3.24, p=.005$, marginal; chest, $t(18)=$ $4.61, p<.001$ ] , and the leg [arm, $t(18)=4.29, p<.001$; chest, $t(18)=5.05, p<.001]$.

Table 1 portrays the actual ranking of body parts by verification times for this experiment (and Experiments $3 \mathrm{~A}$, $3 \mathrm{~B}$, and 4 ) and the body part rankings according to each of the three accounts - part image, distinctiveness, and significance. Because the reaction times for adjacent pairs did not always differ significantly in this and the other experiments, the body parts were placed into three groups for the purpose of comparing the data with the theoretical accounts. The predictions from the three theoretical accounts were also grouped. The head, by virtue of the fact that responses to it was faster than those for almost all the other parts, made up Group 1. The hand, the foot, and the leg times did not differ; therefore, they made up Group 2. Although the arm, the chest, and the back times did differ, they were clearly the parts with the longest verification times and thus made up Group 3.

To provide additional support and a basis for comparison of the theories, 57 Stanford University participants rank ordered the seven body parts by part size, part distinctiveness, or part significance.

The surveys presented the participants with a picture of a human body and asked them to rank the seven body parts according to the specific attribute. The size questionnaire added the following "One way to think about the different body parts is to think about them in terms of their size (i.e., surface area)." The part distinctiveness questionnaire added, "One way to think about the different body parts is to think about them in terms of how much the different parts are distinctive from the rest of the body. Distinctive parts are those which are easily detectable because they are more separate from the other

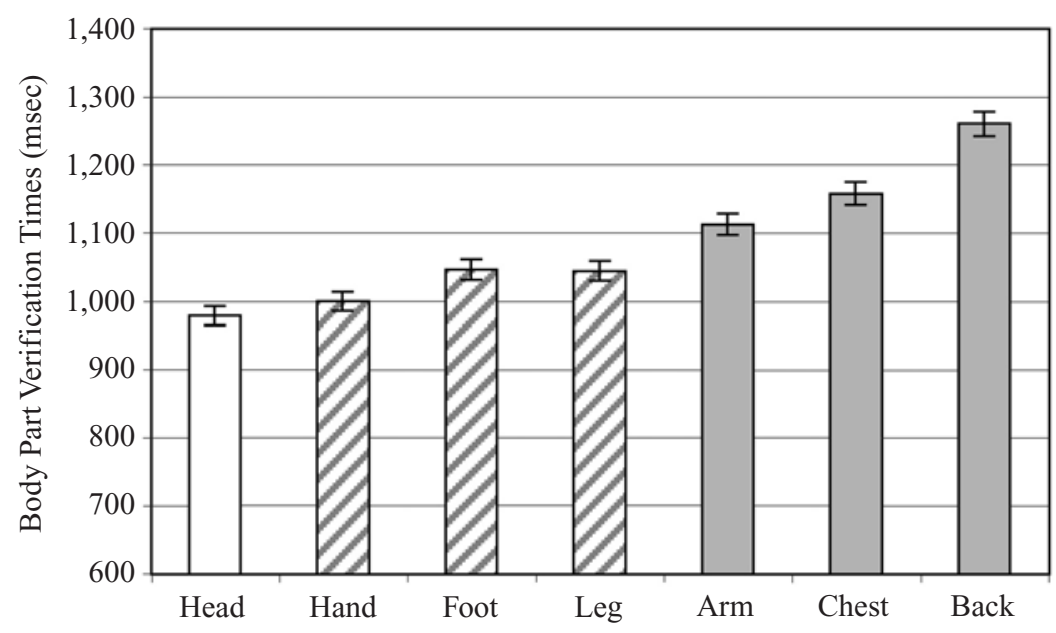

Figure 4. Same body part verification times for the simultaneous body-body part verification experiment. 
Table 1

Data Rankings From Experiment 1 (Simultaneous Body-Body), Experiments 3A and 3B (Name-Whole Body), and Experiment 4 (Sequential Body-Body) and Theoretical Rankings According to Part Size, Part Distinctiveness, and Part Significance

\begin{tabular}{lccccccc}
\hline $\begin{array}{c}\text { Body } \\
\text { Part }\end{array}$ & Exp. 1 & Exp. 3A & Exp. 3B & Exp. 4 & $\begin{array}{c}\text { Part } \\
\text { Size }\end{array}$ & $\begin{array}{c}\text { Part } \\
\text { Distinct. }\end{array}$ & $\begin{array}{c}\text { Part } \\
\text { Signif. }\end{array}$ \\
\hline Head & 1 & 1 & 1 & 1 & 2 & 1 & 1 \\
Hand & 2 & 2 & 2 & 2 & 3 & 1 & 1 \\
Chest & 3 & 2 & 2 & 2 & 1 & 3 & 2 \\
Foot & 2 & 2 & 2 & 2 & 3 & 1 & 1 \\
Arm & 3 & 2 & 3 & 2 & 1 & 2 & 3 \\
Leg & 2 & 3 & 3 & 2 & 1 & 2 & 3 \\
Back & 3 & 3 & 3 & 3 & 1 & 3 & 3 \\
\hline
\end{tabular}

Note-Part size does not account for the data in any experiment, with part significance and part distinctiveness best accounting for the data.

parts. Distinctiveness is not the same as size." The part significance questionnaire added the following, "One way to think about the different body parts is to think about them in terms of their significance. Significance has two components: functionality and distinctiveness. Significant parts are both (1) those that are crucial to the functioning of the organism/object in everyday life and (2) those that are distinctive, which is to say, those that are easily detectable because they are more separate from the other parts (distinctiveness is not the same as size). Consider both these factors together when making your decision."

For part size, the parts were grouped according to physical size, with the largest body parts (the chest, back, leg, and arm) in one group, the medium-size part (the head) in the second group, and the smallest parts (the hand and foot) in the third group. This grouping was confirmed by the survey rankings, which placed the parts in the following order from largest to smallest: the leg $(M=5.44, S D=1.87)$, the back $(M=4.89, S D=$ $1.91)$, the chest $(M=4.44, S D=1.58)$, the arm $(M=$ $4.28, S D=1.13)$, the head $(M=3.89, S D=1.23)$, the foot $(M=2.61, S D=1.75)$, and the hand $(M=2.44$, $S D=2.48)$.

For part distinctiveness, those parts close in distinctiveness were grouped together. Thus, the head, hand, and foot were placed in Group 1, the arm and leg in Group 2, and the chest and back in Group 3. The survey participants ranked distinctiveness in the same way, with the head as most distinctive $(M=5.12, S D=2.45)$, then the hand $(M=4.82, S D=1.55)$, the $\operatorname{arm}(M=4.41$, $S D=1.84)$, the leg $(M=3.82, S D=1.63)$, the foot $(M=3.77, S D=1.25)$, the chest $(M=3.18, S D=$ $2.04)$, and the back $(M=2.77, S D=2.22)$. The only disparity between the reaction time rankings and the judgment rankings was in the placement of the foot. The less distinctive ranking for the foot on the survey was likely a result of the picture of the human body we showed, which did not show the detail of the toes.

For the part significance groupings, with size in the sensorimotor cortex, relative to physical size, as a rough index Group 1 consisted of the head, hand, and foot, Group 2 of the chest, and Group 3 of the arm, leg, and back. For the most part, the questionnaire data support the ordering and the grouping. The head $(M=5.32$, $S D=2.34$ ) was rated the most significant, and the chest was considered quite significant $(M=4.47, S D=2.09)$, higher than the back $(M=3.90, S D=2.16)$, the leg $(M=3.63, S D=1.64)$, the arm $(M=3.42, S D=1.71)$, and the foot $(M=3.05, S D=1.72)$, but also, surprisingly, higher than the hand $(M=3.84, S D=1.89)$. Because part functions are a crucial component to significance, we conducted a second survey in which 60 participants listed the important functions performed by the seven body parts. The instructions stated the following. "For each body part, please list as many of these actions, activities, and responses that you can think of. Some of these parts enclose internal body parts that are also involved with actions and sensations. Please include actions and responses for those enclosed parts of the larger parts as well." The most common functions named were vision (the head), lifting (the arm), holding (the hand), breathing (the chest), support (the back), and walking (the leg and foot). Using a count of listed functions as a proxy for significance, we found that the head had the most functions named across participants (348), followed by the hand (186), the chest (142), the foot (140), the arm (110), the leg (108), and the back (94), which closely approximated our part significance groupings.

To determine the degree of agreement between the response time rankings and the theoretical rankings, Kendall's tau coefficient with ties was calculated. Because there are only seven rankings, the $p$ values have been corrected for continuity. Part size analyses revealed a marginal negative relationship to response time $(\tau=-.62, p=$ .07) with the smaller parts being responded to more quickly than the larger parts. Part distinctiveness best accounts for the data from Experiment $1(\tau=.77, p<.05)$, with part significance marginally related $(\tau=.60, p=$ .07 ). It is important to keep in mind that having only seven body parts and, thus, only seven data points creates difficulty when one tries to find significance. Nevertheless, it is clear that part size does not account for the pattern of data in this body part verification task and that part distinctiveness does a good job of accounting for the pattern of data. 


\section{EXPERIMENT 2 Name-Disembodied Body Part Verification}

The first experiment showed that larger body parts were not verified more quickly than smaller ones. On the contrary, it was the smaller, more significant, and distinctive parts that were verified more quickly. The small white dots as body part cues in the first experiment may have biased against part size. To get around this, in this experiment, the participants compared a named body part with a picture of a disembodied body part, presented sequentially. By presenting disembodied body parts, the size of the part becomes a stronger indicator of the identity of the part. If part size underlies representations of bodies, participants should respond most quickly to the larger parts.

\section{Method}

Participants. Twenty-seven Stanford University undergraduates participated for course credit. Seven participants were removed either because of computer problems or for error rates over $10 \%$. The remaining data from 10 women and 10 men were analyzed.

Stimuli. The 12 poses shown in Figure 1 were modified to include the presentation of a single body part at a time (see Figure 5). Each of the 12 poses was divided into its component parts (head, hand, arm, foot, and leg), preserving the location and the position of each individual part to ensure a precise replication of the stimuli presented in the comparison studies. The chest and the back were eliminated due to the difficulties of recognizing them in isolation.

Design. The design was identical to that in Experiment 1, with the following exceptions. The name of the body part appeared centered on the screen for $1 \mathrm{sec}$, followed by a $500-\mathrm{msec}$ presentation of the fixation point. When the fixation point disappeared, the disembodied body part was displayed and remained on the screen until the participant responded. When ready, the participant initiated the next trial. There was a training block of 15 trials, followed by 245 test trials.

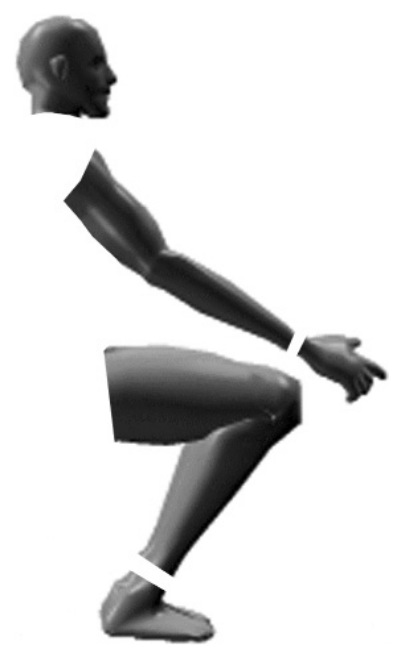

Figure 5. Disembodied body parts. Each of the 12 original body poses was divided into five parts: head, arm, hand, leg, and foot. The parts were shown at the same screen locations at which they would have appeared as parts of the whole body. The parts were rotated $0^{\circ}, 90^{\circ}$, or $180^{\circ}$ from upright.
Due to a software design problem, the testing stimuli had to be presented in a fixed order. To deal with possible order effects, four random orders of the stimuli were created, with the constraint that the same pose, the same named part, or the same cued part could not appear more than three times in a row. No orientation appeared more than four consecutive times.

Equipment and Procedure. The equipment was identical to that used in Experiment 1. The instructions were altered to reflect the nature of the new task. The participants spent approximately 40 min completing the task.

\section{Results and Discussion}

We performed a two-way repeated measures ANOVA on same responses with the within-subjects factors of body part (arm, foot, hand, head, or leg) and orientation of the presented body part $\left(0^{\circ}, 90^{\circ}, 180^{\circ}\right)$. The participants responded most quickly to body parts in the $0^{\circ}$ $(M=733 \mathrm{msec}, S D=325)$ orientation $[F(2,38)=5.89$, $\left.M S_{\mathrm{e}}=12,067, p<.01\right]$. Those in the $90^{\circ}(M=790 \mathrm{msec}$, $S D=389)$ and the $180^{\circ}(M=771 \mathrm{msec}, S D=347)$ orientations did not differ $\left[0^{\circ}\right.$ vs. $90^{\circ}, t(19)=3.38, p<.01$; $0^{\circ}$ vs. $180^{\circ}, t(19)=3.65, p<.01 ; 90^{\circ}$ vs. $180^{\circ}, t(19)=$ $0.72, p=.48]$.

Verification times were shortest for the head $(M=$ $631 \mathrm{msec}, S D=283)$, followed by the hand $(M=$ $662 \mathrm{msec}, S D=239)$, the arm $(M=818 \mathrm{msec}, S D=$ $375)$, the foot $(M=856 \mathrm{msec}, S D=356)$, and the leg $(M=876 \mathrm{msec}, S D=430)\left[F(4,76)=18.27, M S_{\mathrm{e}}=\right.$ $41,382, p<.01$, see Figure 6]. The verification times for the head were shorter than those for each of the other parts [arm, $t(19)=4.14, p=.001$; foot, $t(19)=9.57$, $p<.001$; hand, $t(19)=3.67, p=.002$; leg, $t(19)=$ $5.07, p<.001]$. The foot and the leg times were longer than those for both the head and the hand [foot, $t(19)=$ 9.48, $p<.001$; leg, $t(19)=4.30, p<.001]$, with the arm times marginally slower $[t(19)=3.28, p=.004]$. There was no interaction of orientation and body part $\left[F(8,152)=1.21, M S_{\mathrm{e}}=8,547, p=.30\right]$.

Although the times generally followed the pattern in the first experiment, the verification times for the foot were longer than expected. Of the five body parts used in this experiment, the foot, when disembodied, looked least like the part it was meant to resemble, as can be seen in Figure 5. As a result, the longer verification time may reflect a two-part process of recognition. First, the participant recognizes the part as small - thus, either the hand or the foot. Second, because of the lack of distinctiveness of the part, the participant spends more time in making the foot judgment.

As in Experiment 1, parts high in distinctiveness and significance were verified more quickly. Large body parts were actually verified more slowly than small ones, even when the parts were disembodied, providing additional evidence against the part size account. Because the chest and the back were not included, part distinctiveness and part significance cannot be separated. When visual depictions of human body parts are viewed, larger size does not lead participants to verify the match between a named and a depicted part more quickly. Instead, 


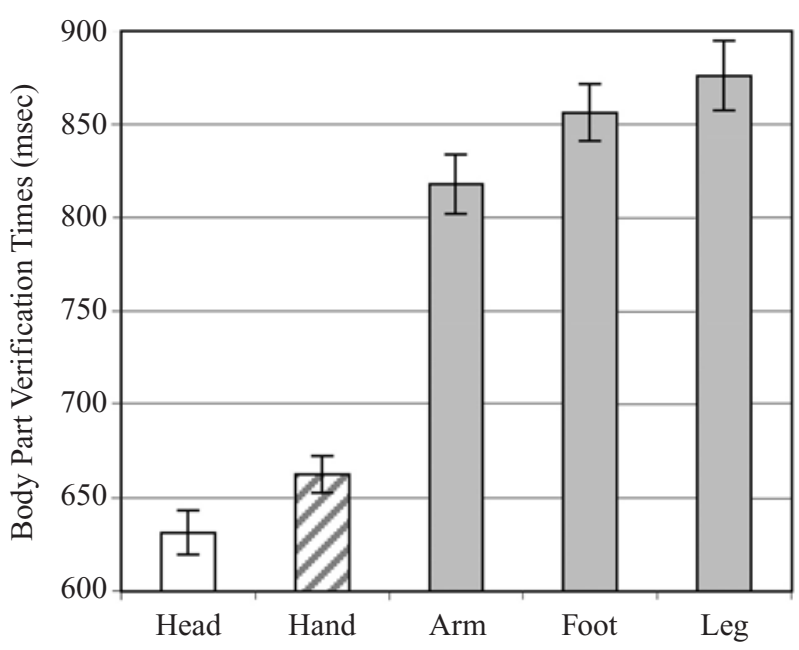

Figure 6. Same body part verification times for the namedisembodied body part verification experiment.

participants make their judgments on the basis of the part's visual distinctiveness and functional significance.

\section{EXPERIMENT 3A Name-Whole Body Part Verification}

In two experiments, the participants were faster to verify depicted body parts that were distinctive and significant, rather than large. The first experiment was strictly visual, and although the second one used named body parts, it did not use parts that separate the distinctiveness and the significance accounts. Here, the participants saw one of seven body part names - head, arm, hand, chest, back, leg, or foot - followed by a full-body depiction with one of those parts highlighted. What effect will naming have for full-body verification times? Names for body parts are frequent, familiar, and used from an early age. They are also metaphorically extended, on the basis of both shape and function, as in the arm of a chair or the head of a committee. Names, then, may evoke knowledge about the behavior and function of body parts more than visual presentation does. There is some support for the hypothesis that naming evokes functional considerations over and above depiction. In a task requiring segmenting films of every day activities, such as doing the dishes, into coarse- and fine-level units, Zacks, Tversky, and Iyer (2001) found greater hierarchical organization when observers described the action of each unit while segmenting. The descriptions - typically, actions on objectscalled attention to top-down functional information. If functional information about the body parts arises from naming, verification times may reflect the significance of the parts.

\section{Method}

Participants. Twenty-four Stanford University undergraduates participated for course credit. The data from 4 participants were discarded, 1 due to a computer error during testing and 3 due to error rates greater than $10 \%$. The results from 11 women and 9 men were analyzed.

Stimuli. The 12 poses shown in Figure 1 were used as stimuli. The combinations of cued body part, pose, and orientation were identical to those described in Experiment 1.

Design, Equipment, and Procedure. The design differed from that in Experiment 2 in three ways. First, following the name of the part and the fixation point, the participants saw a whole body with a part cued, instead of a disembodied part. Second, all seven body parts were cued. Third, the participants engaged in 20 practice trials and 420 test trials. The equipment and the procedure were the same as those in Experiment 2. The participants spent approximately $1 \mathrm{~h}$ completing the experiment.

\section{Results and Discussion}

As before, we performed a two-way repeated measures ANOVA with within-subjects factors of body part (arm, back, chest, foot, hand, head, or leg) and orientation of the presented body part $\left(0^{\circ}, 90^{\circ}\right.$, or $\left.180^{\circ}\right)$. The participants responded most quickly when the pose was at $0^{\circ}(M=780 \mathrm{msec}, S D=362)$ and responded more slowly when it was at $90^{\circ}(M=810 \mathrm{msec}, S D=357)$ or $180^{\circ}(M=809 \mathrm{msec}, S D=340)$, which did not differ $\left[F(2,38)=8.21, M S_{\mathrm{e}}=4,746, p<.01 ; 0^{\circ}\right.$ vs. $90^{\circ}, t(19)=$ $4.22, p<.01 ; 0^{\circ}$ vs. $180^{\circ}, t(19)=2.82, p<.05 ; 90^{\circ}$ vs. $\left.180^{\circ}, t(19)=0.04, p=.97\right]$.

Response times for each body part appear in Figure 7. Speed differed by part $\left[F(6,114)=11.47, M S_{\mathrm{e}}=8,506\right.$, $p<.01]$, with the shortest times to the head $(M=$ $723 \mathrm{msec}, S D=312)$, followed by the hand $(M=$ $789 \mathrm{msec}, S D=257)$, the chest $(M=780 \mathrm{msec}, S D=$ $320)$, the foot $(M=800 \mathrm{msec}, S D=344)$, the $\operatorname{arm}(M=$ $811 \mathrm{msec}, S D=345)$, the leg $(M=827 \mathrm{msec}, S D=$ $264)$, and the back $(M=864 \mathrm{msec}, S D=410)$. Paired $t$ tests revealed that verification times were shorter to the head than to any of the other parts, except the hand [arm, $t(19)=5.97, p<.001$; back, $t(19)=4.98, p<.001$; chest, $t(19)=3.58, p=.002$; foot, $t(19)=3.49, p=$ $.002 ;$ leg, $t(19)=5.93, p<.001)$. Verification times for the leg were longer than the times for the head and the hand $[t(19)=5.07, p<.001]$. Times for the back were longer than the times for the head, the hand $[t(19)=3.97$, $p=.001]$, and the chest $[t(19)=3.80, p=.001]$. Orientation did not interact with body part $[F(12,228)=$ $\left.1.15, M S_{\mathrm{e}}=5,482, p=.32\right]$.

In order to compare the results of this experiment with those of earlier experiments, the verification rankings were divided into groups. Table 1 depicts these rankings, as well as the rankings from Experiments 1, 3B, and 4, and the rankings according to each of the three accountspart image, distinctiveness, and significance. The data fit part significance best, with a significant correlation $(\tau=.69, p<.05)$. Although times increased with decreases in part distinctiveness, the correlation was not significant ( $\tau=.53, p=.10$ ). There was a negative, although not significant, relationship between verification times and part size $(\tau=-.41, p=.17)$. Quantitatively, the main difference between the pattern of reaction times with 


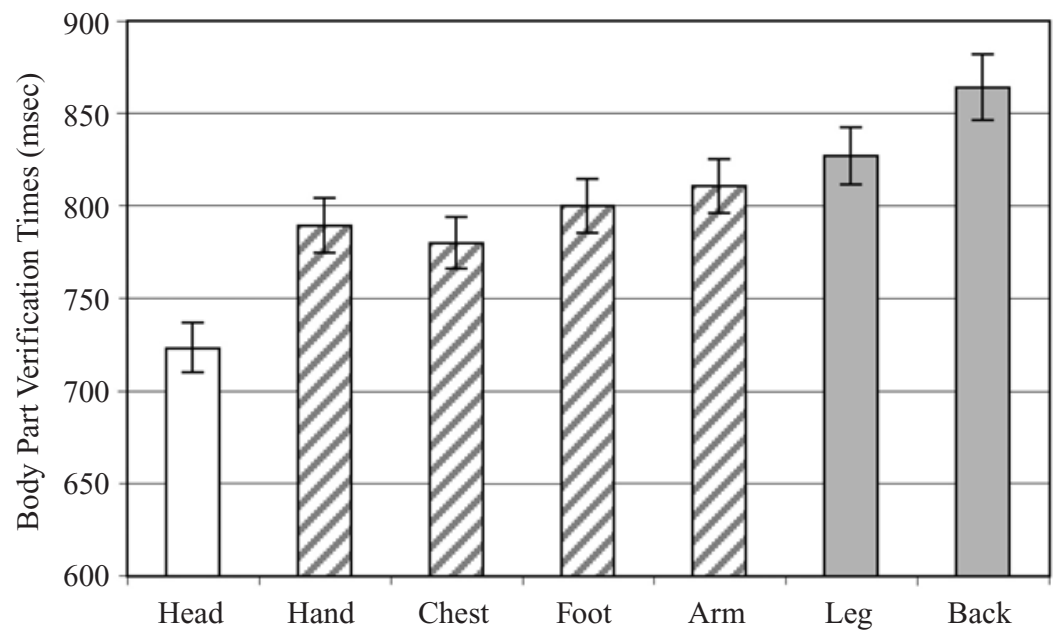

Figure 7. Same body part verification times for the name-whole body part verification experiment.

name-body comparisons and that with body-body comparisons is that responses for the chest are relatively fast for name-body comparisons. As analyzed earlier, the chest, although not distinctive, is significant. Altogether, then, the evidence suggests that part significance is a more important determinant of the body schema when the parts are invoked by language than part distinctiveness is.

\section{EXPERIMENT 3B \\ Name-Whole Body Part Verification Replication}

Because of the subtlety of the change in the ordering of the chest body part, we decided to replicate the namebody experiment. At the same time, a new version of Fractal Design Poser (1996) allowed creating bodies with more realistic shading and coloring.

\section{Method}

Participants. Thirty-four Stanford University students were paid for their participation in this experiment. The data from 1 participant was discarded due to an error rate above $10 \%$. The data from 20 women and 13 men were analyzed.

Stimuli. The poses used in Experiment 3A were re-created using Fractal Design Poser (1996). The same small white dot was used to cue the seven body parts. However, because of the more natural and lighter coloring of the bodies, the circle had a black edge, to clearly distinguish it from the rest of the body.

Design. The design differed from that in Experiment 3A in that all the poses were shown upright, at $0^{\circ}$ orientation. There were no $90^{\circ}$ or $180^{\circ}$ rotations of the poses. With the removal of the rotated poses, the number of trials was reduced from 420 to 140 . The number of training trials (20) remained the same, with previously rotated poses replaced by upright poses.

Equipment and Procedure. The same equipment and instructions were used in this experiment as in those Experiment 3A. Procedural changes included showing the named body part for $1.5 \mathrm{sec}$, and using a single version of the experimental program to randomize and present the test trials. The participants completed the experiment within $20 \mathrm{~min}$.

\section{Results and Discussion}

We performed a one-way repeated measures ANOVA with a within-subjects factor of body part (arm, back, chest, foot, hand, head, or leg) on same responses. Figure 8 shows that the participants responded to the named and the cued body parts in the same order as that in Experiment $3 \mathrm{~A}$-namely, the head $(M=650 \mathrm{msec}, S D=$ $259)$, the hand $(M=675 \mathrm{msec}, S D=206)$, the chest $(M=708 \mathrm{msec}, S D=286)$, the foot $(M=710 \mathrm{msec}$, $S D=236)$, the $\operatorname{arm}(M=726 \mathrm{msec}, S D=253)$, the leg $(M=751 \mathrm{msec}, S D=240)$, and the back $(M=767 \mathrm{msec}$, $S D=276)\left[F(6,192)=15.61, M S_{\mathrm{e}}=3,459, p<.01\right]$. Paired $t$ tests revealed differences between body parts that were nearly identical to those in the earlier experiment. Head response times were shorter than those to any other part $[\operatorname{arm}, t(32)=6.25, p<.001$; back, $t(32)=$ $7.26, p<.001$; chest, $t(32)=4.00, p<.001$; foot, $t(32)=$ $3.92, p<.001$; hand, $t(32)=3.63, p=.001$; leg, $t(32)=$ 6.66, $p<.001]$. Arm response times were longer than hand $[t(32)=4.73, p<.001]$ and head times. Leg response times were longer than hand $[t(32)=4.84, p<$ $.001]$ and head times. Back response times were longer than chest $[t(32)=3.68, p=.001]$, hand $[t(32)=5.38$, $p<.001]$, and head times and were marginally longer than foot times $[t(32)=3.12, p=.004]$. Just as with Experiments 1 and $3 \mathrm{~A}$, Table 1 shows the body part rankings for this experiment. Part significance is the best predictor of verification times $(\tau=.69, p<.05)$, with part distinctiveness positively related but not significant ( $\tau=$ $.53, p=.10)$. Part size is negatively related to verification times $(\tau=-.53, p=.10)$.

Experiment $3 \mathrm{~B}$ provided additional evidence for the part significance account of verification times when naming is involved. As with Experiment 3A, the ordering of the verification times for the body parts corresponded best both qualitatively and quantitatively to the 


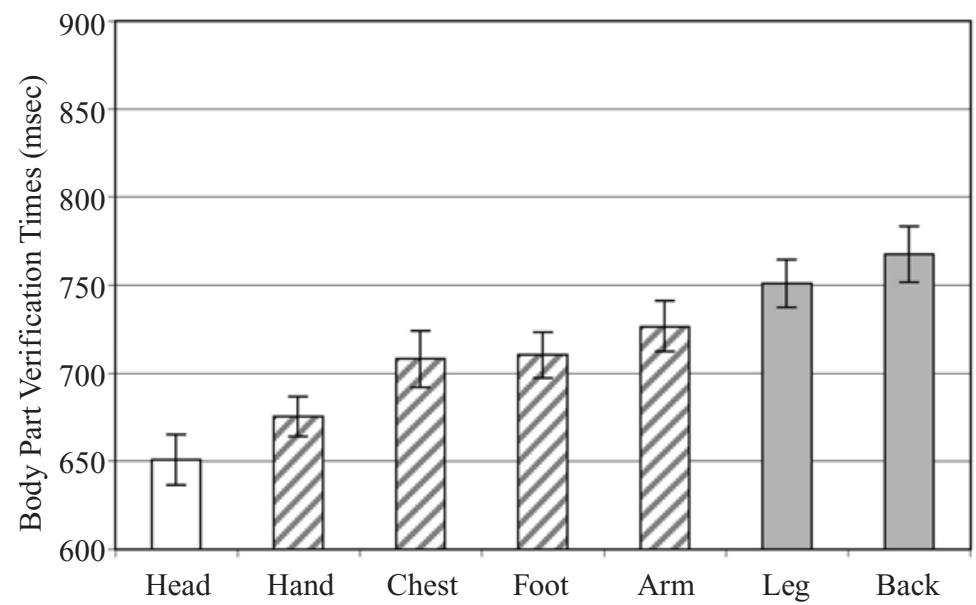

Figure 8. Same body part verification times for the name-whole body part verification replication experiment.

predictions of the part significance account. These results add support to the idea that names evoke function more than visual presentation does.

\section{EXPERIMENT 4 Sequential Body-Body Part Verification}

The part size account of body part verification times has failed in four experiments, one in which pictures of bodies were directly compared and three in which names of body parts were compared with disembodied parts or highlighted parts in pictures of bodies. The body-body verification times corresponded best to the part distinctiveness account, according to which parts with high contour distinctiveness are more readily verified. The name-body verification times corresponded best to the part significance account, by which parts that are perceptually salient and functionally significant are most readily verified. Part distinctiveness and part significance are highly correlated, although separable when function deviates from salience. Names, because they are abstract, are more likely to evoke function than visual stimuli do, so it makes sense that part significance appears to govern verification times when named parts are compared with pictures of bodies.

When equivalent stimuli are presented as pictures or words, they are sometimes transformed to the other medium, depending on task demands, especially timing (e.g., Posner, 1969; Posner \& Keele, 1968; Tversky, 1969, $1974,1975)$. When one kind of match is more efficacious than the other and the task allows transformation of modality, participants often transform. Will the part significance pattern emerge for pairs of pictures of bodies if conditions encourage participants to implicitly name the body parts?

In Experiment 1, the participants judged the match between cued parts on two simultaneously depicted bodies.
Here, the participants completed the same task, except that the depictions were presented sequentially, with $2 \mathrm{sec}$ between them, allowing and encouraging transformation of depictions into words by presenting the depicted bodies sequentially. This imposed a memory load in the participants. Instead of retaining the picture of the entire body with the appropriate part highlighted, the participants could reduce the memory load by simply retaining the name of the highlighted part and comparing that with the part depicted in a second picture. If the participants implicitly named, part significance should account for the verification times better than part distinctiveness, even though both stimuli are visual. Numerous previous studies have shown that visual stimuli are transformed to verbal ones when a memory load is imposed (e.g., Posner \& Keele, 1968).

\section{Method}

Participants. Seventy Stanford University students were paid or received course credit as compensation for their participation in this experiment. The data from 9 participants were eliminated because of computer error or participant error rates greater than $10 \%$, leaving the data from 36 women and 25 men for analysis.

Stimuli. The body poses re-created with a new version of Fractal Design Poser (1996) for Experiment 3B were used in this experiment. The combinations of cued body part, pose, and orientation were identical to those used in Experiment 1. The stand and squat poses were eliminated, due to their redundancy with other poses and to shorten the length of the experiment.

Equipment, Design, and Procedure. The same equipment was used in this experiment as in the previous experiments. The structure of the experiment was similar to that in Experiment 3A. A picture of a body with a part cued, instead of a part name, was shown for $1 \mathrm{sec}$. After a 1.5-sec delay, a fixation point appeared for $500 \mathrm{msec}$, followed by the picture of the second body with a part cued. This second picture disappeared when the participant responded. Adding the additional 1.5-sec delay significantly increased the length of the experiment but was important in encouraging the participants to implicitly name the body part cued first. Therefore, each participant saw only half of all possible trials, 168 instead of 336 . The tri- 
als were randomly assigned to each participant. The participants completed the experiment within $30 \mathrm{~min}$.

\section{Results and Discussion}

We performed a two-way repeated measures ANOVA on same responses, with within-subjects factors of body part (arm, back, chest, foot, hand, head, or leg) and orientation disparity $\left(90^{\circ}\right.$ or $\left.180^{\circ}\right)$. When verifying whether the two cued body parts were the same, the participants were faster to respond when the poses were displaced by $90^{\circ}(M=946 \mathrm{msec}, S D=360)$ than when they were displayed by $180^{\circ}[M=965 \mathrm{msec}, S D=369, F(1,56)=$ $\left.7.08, M S_{\mathrm{e}}=21,050, p<.05\right]$. Orientation did not interact with body part $\left[F(6,336)=1.16, M S_{\mathrm{e}}=18,069, p=\right.$ .33].

The pattern of data in this experiment was nearly identical to that in the experiments in which body parts were named-Experiment 2, 3A, and 3B. Specifically, the ordering of body parts from shortest verification times to longest was the head $(M=897 \mathrm{msec}, S D=366)$, the hand $(M=948 \mathrm{msec}, S D=375)$, the chest $(M=941 \mathrm{msec}$, $S D=359)$, the foot $(M=946 \mathrm{msec}, S D=373)$, the arm $(M=956 \mathrm{msec}, S D=335)$, the leg $(M=968 \mathrm{msec}$, $S D=358)$, and the back $(M=1,018 \mathrm{msec}, S D=363)$ $\left[F(6,336)=6.09, M S_{\mathrm{e}}=19,375, p<.01\right.$; see Figure 9]. Paired $t$ tests revealed that verification times for the head were shorter than those for all the other parts, except the chest $[$ arm, $t(60)=4.85, p<.001$; back, $t(60)=6.41$, $p<.001$; foot, $t(60)=3.73, p<.001$; hand, $t(60)=$ $3.40, p=.001$; leg, $t(60)=4.17, p<.001)$, whereas the times for the back were longer than those for the head, the chest $[t(60)=3.37, p=.001]$, the foot $[t(60)=3.33$, $p=.001]$, and the hand $[t(60)=3.45, p=.001]$ and were marginally longer than those for the leg $[t(60)=$ $3.11, p=.003]$ and the arm $[t(60)=2.92, p=.005]$. Notably, responses for the chest, which is low in part distinctiveness but relatively higher in significance, were relatively fast here, in contrast to the case of simultaneous presentation of depicted bodies. Table 1 shows the rankings for the body parts on the basis of verification times. Despite the similarity between the ordering of times in this experiment and in the previous naming experiments, none of the three theoretical accounts significantly predicted the verification times. As in all the other experiments, larger parts were not verified more quickly than smaller parts $(\tau=-.27, p=.29)$. Part significance and part distinctiveness were marginal in predicting verification times $(\tau=.54, p=.10$, and $\tau=.60$, $p=.08$, respectively).

Once again, part size fails to predict verification times when participants make judgments about depictions of human bodies with parts cued. Participants respond most quickly to the more significant and distinctive parts, rather than to the largest parts. Because there is very little variability among the verification times for the hand, the chest, the foot, the arm, and the leg, the tau analyses do not reveal a best fit with either part significance or part distinctiveness. However, the pattern of the data replicates more precisely the pattern of the name-body comparisons (Experiments $3 \mathrm{~A}$ and $3 \mathrm{~B}$ ) than it does the pattern of the simultaneous body-body comparisons (Experiment 1). The primary difference between these data and those in Experiment 1 is the rank order of the chest body part. The chest, which has functional and behavioral significance, is not a distinctive part. When body parts are named explicitly, as in Experiments 3A and $3 \mathrm{~B}$, or implicitly, as in the present experiment, the verification time ranking of the parts is best accounted for by an explanation involving the significance of the body parts. In this case, the chest is more quickly verified than its most visually salient comparable part, the back. When the body parts are not named, participants can respond to the depicted bodies as purely visual objects. When only perception is involved, part verification

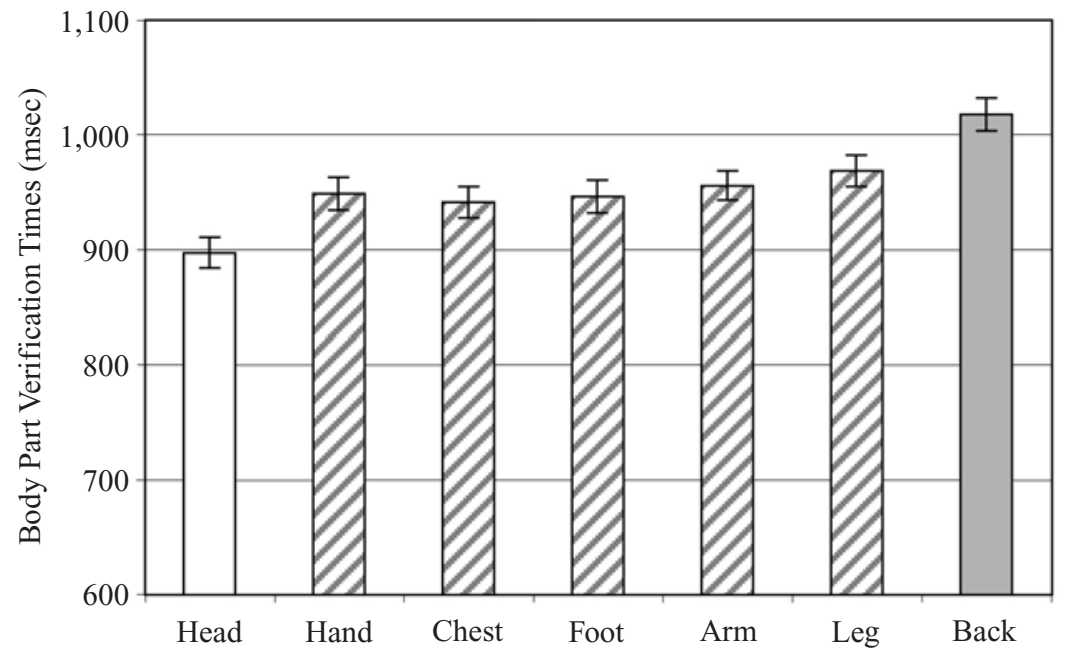

Figure 9. Same body part verification times for the sequential body-body part verification experiment. 
is best when based on the distinctiveness of the body parts, evidenced by the longer response time to the chest. The present results add support to two claims: that the conditions of the experiment encourage implicit naming, and that naming favors functional features of bodies, as well as perceptual ones.

\section{GENERAL DISCUSSION}

People experience the human body both from the inside and from the outside. Insider knowledge of the body provides a wealth of sensory information, some of it in response to action. It also includes direct knowledge of intention and the roles various parts of the body have in carrying out intentions. Outsider information is the same for bodies as for other objects: information about appearancein particular, shape. Research reviewed earlier, in which patients, brain imagery, and normal participants were used in a variety of tasks, supports the theoretical claim that knowledge of the body is privileged. The research reported here corroborates and expands that claim. The situation investigated was body part verification of those parts commonly named across languages: head, arm, hand, chest, back, leg, and foot. Three accounts of reaction times make different predictions regarding the verification of body parts. According to the part size account, derived from the classic work on imagery (e.g., Kosslyn, 1980), larger parts should be more readily detected in perception than smaller ones, just as they are in imagery. According to the part distinctiveness account, which extends research on object recognition (e.g., Biederman, 1987; Hoffman \& Richards, 1984), parts with greater contour discontinuity should be detected more quickly. Both these accounts rely only on visual information. The third account, part significance, based on work on categorization (Tversky \& Hemenway, 1984), combines visual information, as well as the behavior or function of body parts. Supporting this account is the fact that relative area of projection to the sensorimotor cortex is only weakly correlated with part size. Rather, relative area in the sensorimotor cortex roughly corresponds to part significance, presumably because more functional parts need finer motor control and finer sensory feedback. Significant parts tend to be high in distinctiveness, but the relationship is not perfect.

For all verification tasks - those between depicted bodies and those between named parts and depictionsthe size of the body part was inversely related to speed of verification. That is, smaller parts were, in fact, verified more quickly than larger parts, refuting the part size account.

Despite the similarities between the part significance and the part distinctiveness accounts, the data presented here provide preliminary evidence for a partial dissociation between the two accounts. When comparisons were conceptual - that is, when a named body part was compared with a depicted one, as in Experiments 3A and $3 \mathrm{~B}$ - the part significance account best predicted the speed with which body parts were verified. In contrast, when comparisons were perceptual - that is, when two depicted bodies were compared-part distinctiveness best accounted for the response times. The interpretation we offer for these findings is that naming evokes function, the element that the significance account incorporates.

In addition to the correlations between the verification times and the theoretical accounts, evidence supporting the claim that naming evokes function comes from the pattern of reaction times - specifically, the times associated with the chest and the back. Chests, although not visually distinct, are functionally important, more so than backs. In accordance with this view, the participants listed more functions for the chest than for the back (2.3 for the chest and 1.6 for the back, on average, for 159 participants). Chests house and protect numerous internal organs, whereas backs are viewed simply as support for the body. Nearly a dozen experiments on the space surrounding the body have shown that fronts were more accessible than backs as cues to locations around the body (Bryant, Tversky, \& Franklin, 1992; Franklin \& Tversky, 1990). The superiority of the front, they argued, derives from the fact that the front refers to the world that can be easily perceived and manipulated, the world of navigation and action, in contrast to the back. An entirely different paradigm, interpreting a letter "sketched" on the body, also showed a strong bias for the front of the body (Parsons \& Shimojo, 1987).

These disparate lines of evidence converge to suggest that chests have greater functional significance than backs do, although they are comparable in perceptual distinctiveness. In the experiments in which the body parts were explicitly or implicitly named (3A, 3B, and 4 ), chests were responded to more quickly than backs, supporting the view that the participants responded on the basis of part significance more often than on the basis of part distinctiveness. In Experiment 1, in which the two bodies were presented simultaneously, the chest and the back were verified equally slowly, supporting part distinctiveness.

The part significance pattern appeared in two tasks: in name-body comparisons and in sequential body-body comparisons. Considerable previous research has shown that visual stimuli are often converted to names when memory is required (e.g., Posner \& Keele, 1968). The present finding replicates that and also demonstrates that implicit naming, like explicit naming, serves to emphasize functional aspects of concepts - in this case, body parts.

Why should significance predict body part verification better when explicit or implicit naming is entailed, but distinctiveness predict better when bodies are compared simultaneously? When two bodies appear together on the screen and the task is to say whether the cue appears on the same or on different parts, the bodies seem to be perceived just like any other object-as visual forms, shapes with part boundaries suggested by contour discontinuities. Searching for the cues and comparing 
across objects does not require any cognizance of the objects or the parts. When a named part is compared with a cued part, the name itself must be comprehended and transformed into an expectation of a subshape bearing a constrained spatial relation to the whole. This comprehension and translation seems to activate functional features, in addition to perceptual ones. When body parts are named, then depicted on whole bodies or alone, or when the pictured bodies are separated in time to encourage implicit naming of the part cued first, part verification speed seems to depend on mental representations of the body that reflect the functions of the body, as well as the appearance. Converging research has shown the significance of names in arousing functional abstract properties of concepts. Naming directs children to superordinate, rather than thematic, relations among objects (Markman \& Hutchinson, 1984). Describing visual depictions of everyday events focuses observers on the goal structures of those events (Zacks, Tversky, \& Iyer, 2001).

Invoking the name of a body part accesses a mental representation of the parts of the body that is based not only on the appearance of the body, but also on the behavior and function of the body. This special link between the naming of body parts and our knowledge of them likely comes from our internal experience of our bodies. This is an experience we have of no other object in the world, and for that reason, if for no other, bodies are special. What is impressive about the human mind is that a purely symbolic stimulus, a name, can elicit both perceptual and abstract information and can access the abstract more readily than can depictions.

\section{REFERENCES}

ANDERSEN, E. S. (1978). Lexical universals of body-part terminology. In J. H. Greenberg (Ed.), Universals of human language (Vol. 3, pp. 335-368). Stanford, CA: Stanford University Press.

BIEDERMAN, I. (1987). Recognition-by-components: A theory of human image understanding. Psychological Review, 94, 115-147.

BRown, C. H. (1976). General principles of human anatomical partonomy and speculations on the growth of partonomic nomenclature. American Ethnologist, 3, 400-424.

Bryant, D. J., Tversky, B., \& Franklin, N. (1992). Internal and external spatial frameworks for representing described scenes. Journal of Memory \& Language, 31, 74-98.

BURTON, M. L., \& KIRK, L. (1979). Ethnoclassification of body parts: A three-culture study. Anthropological Linguistics, 21, 379-399.

Carpenter, P. A., \& Just, M. A. (1978). Eye fixations during mental rotation. In J. W. Senders, D. F. Fisher, \& R. A. Monty (Eds.), Eye movements and the higher psychological functions (pp. 115-133). Hillsdale, NJ: Erlbaum.

Chatterjee, S. H., Freyd, J. J., \& Shiffrar, M. (1996). Configural processing in the perception of apparent biological motion. Journal of Experimental Psychology: Human Perception \& Performance, 22, 916-929.

COOPER, L. A. (1975). Mental rotation of random two-dimensional shapes. Cognitive Psychology, 7, 20-43.

CoOper, L. A., \& SHEPARD, R. N. (1973). Chronometric studies of the rotation of mental images. In W. G. Chase (Ed.), Visual information processing (pp. 75-176). San Diego: Academic Press.

Downing, P., Jiang, Y., Shuman, M., \& Kanwisher, N. (2001). A cor- tical area selective for visual processing of the human body. Science, 293, 2470-2473.

Fractal Design Poser (Version 1.0) [Computer software] (1995). Aptos, CA: Fractal Design Corporation.

Fractal Design Poser (Version 2.0) [Computer software] (1996). Aptos, CA: Fractal Design Corporation.

Franklin, N., \& TVErsky, B. (1990). Searching imagined environments. Journal of Experimental Psychology: General, 119, 63-76.

Goodnow, J. (1977). Children drawing. Cambridge, MA: Harvard University Press.

Gross, C. G., \& Graziano, M. S. A. (1995). Multiple representations of space in the brain. Neuroscientist, 1, 43-50.

Guariglia, C., \& AntonucCI, G. (1992). Personal and extrapersonal space: A case of neglect dissociation. Neuropsychologia, 30, 10011009.

Hoffman, D. D., \& Richards, W. A. (1984). Parts of recognition. Cognition, 18, 65-96.

KellogG, R. (1969). Analyzing children's art. Palo Alto, CA: National Press.

KossLYN, S. M. (1976). Can imagery be distinguished from other forms of internal representation? Evidence from studies of information retrieval times. Memory \& Cognition, 4, 291-297.

Kosslyn, S. M. (1980). Image and mind. Cambridge, MA: Harvard University Press.

Markman, E. M., \& Hutchinson, J. E. (1984). Children's sensitivity to constraints on word meaning: Taxonomic versus thematic relations. Cognitive Psychology, 16, 1-27.

McMulLEN, P. A., \& JoliCGEUR, P. (1992). Reference frame and effects of orientation on finding the tops of rotated objects. Journal of Experimental Psychology: Human Perception \& Performance, 18, 807820.

Metzler, J. (1973). Cognitive analogues of the rotation of threedimensional objects. Unpublished doctoral dissertation, Stanford University.

PARSONS, L. M. (1987). Imagined spatial transformations of one's body. Journal of Experimental Psychology: General, 116, 172-191.

PARSONS, L. M. (1990). Body image. In M. W. Eysenck (Ed.), The Blackwell dictionary of cognitive psychology (p. 46). Oxford: Blackwell.

PARsons, L. M. (1994). Temporal and kinematic properties of motor behavior reflected in mentally simulated action. Journal of Experimental Psychology: Human Perception \& Performance, 20, 709-730.

PARSONS, L. M., \& SHIMOJO, S. (1987). Perceived spatial organization of cutaneous patterns on surfaces of the human body in various positions. Journal of Experimental Psychology: Human Perception \& Performance, 13, 488-504.

PENFIELD, W., \& RASMUSSEN, T. (1950). The cerebral cortex of man. New York: Macmillan.

Posner, M. I. (1969). Abstraction and the process of recognition. In G. H. Bower \& J. T. Spence (Eds.), The psychology of learning and motivation (Vol. 3, pp. 44-100). New York: Academic Press.

Posner, M. I., \& Keele, S. W. (1968). On the genesis of abstract ideas. Journal of Experimental Psychology, 77, 353-363.

PsYSCOPE (Version 1.0) [Computer software] (1994). Pittsburgh: Carnegie Mellon University.

ReED, C. L., \& FARAH, M. J. (1995). The psychological reality of the body schema: A test with normal participants. Journal of Experimental Psychology: Human Perception \& Performance, 21, 334-343.

RoTH, M. (1949). Disorders of the body image caused by lesions of the right parietal lobe. Brain, 72, 89-111.

Shiffrar, M., \& FreYD, J. J. (1990). Apparent motion of the human body. Psychological Science, 1, 257-264.

TARR, M. J., \& PINKER, S. (1991). Orientation-dependent mechanism in shape recognition: Further issues. Psychological Science, 2, 207-209.

TVERSKY, B. (1969). Pictorial and verbal encoding in a short-term memory task. Perception \& Psychophysics, 6, 225-233.

TVERsKy, B. (1974). Retrieval of pictorial and verbal stimulus codes. Bulletin of the Psychonomic Society, 4, 580-582.

Tversky, B. (1975). Pictorial encoding in sentence-picture com- 
parison. Quarterly Journal of Experimental Psychology, 27, 405410.

Tversky, B., \& Hemenway, K. (1984). Objects, parts, and categories. Journal of Experimental Psychology: General, 113, 169-193.

WEINSTEIN, S. (1968). Intensive and extensive aspects of tactile sensitivity as a function of body part, sex, and laterality. In D. R. Kenshalo (Ed.), The skin senses (pp. 195-222). Springfield, IL: Thomas.

Zacks, J. M., Mires, J., Tversky, B., \& Hazeltine, E. (2000). Mental spatial transformations of objects and perspective. Journal of Spatial Cognition \& Computation, 2, 315-332.
Zacks, J. M., Ollinger, J. M., Sheridan, M., \& Tversky, B. (2002). A parametric study of mental spatial transformations of bodies. NeuroImage, 16, 857-872.

ZACKS, J. M., TVERSKY, B., \& Iyer, G. (2001). Perceiving, remembering, and communicating structure in events. Journal of Experimental Psychology: General, 130, 29-58.

(Manuscript received August 21, 2002; revision accepted for publication June 29, 2004.) 